\title{
Chemistry: the creative force
}

\section{3rd EuCheMS Chemistry Congress in Nuremberg (Germany)}

\author{
Steffen Pauly
}

Received: 20 October 2010/ Accepted: 21 October 2010/Published online: 9 November 2010

(C) Springer-Verlag 2010

The brand-new congress center in the medieval city of Nuremberg, Germany was the venue for the 3rd EuCheMS Chemistry Congress in September 2010. It attracted roughly 2500 scientists from 60 different countries. Organized under the auspices of the European Association for Chemical and Molecular Sciences (EuCheMS) by the German Chemical Society (GDCh), this event followed the EuCheMS Chemistry Congresses in Budapest (2006) and Torino (2008). It seems that this series has now established itself as Europe's largest and most outstanding showcase for chemistry.

Seven main themes, each hosting three or four symposia, were at the center of the congress. These included "innovative materials," "resources and environment," "supramolecular systems," "catalysis," "molecular life sciences," "analysis, manipulation, and simulation," and "advances in organic and inorganic chemistry." These themes were broadly introduced by seven plenary speakers and discussed in great detail in the symposia, which featured invited lectures and poster sessions. The main themes were selected for their significance in terms of future impact. "Chemistry generates much of its progress and achievements for a sustainable society within these seven areas," explained Andreas Hirsch, Co-chair of the Scientific Committee.

The seven plenary lectures were held by outstanding scientists from all over the world. Barbara Imperiali (Cambridge, MA, USA) presented two multidisciplinary initiatives focused on the development of novel probes for the study of complex biological systems in her lecture on "Fluorescent Tools for Chemical Biology." Klaus Müllen

S. Pauly $(\bowtie)$

Springer, Tiergartenstrasse 17, 69121 Heidelberg, Germany

e-mail: steffen.pauly@springer.com
(Mainz, Germany) addressed "Macromolecules, Assemblies, Particles-A Discovery Journey in Materials Synthesis" and demonstrated the power of synthesis to obtain new functional nanoparticles. "Molecular Photovoltaics" was the theme of Michael Grätzel (Lausanne, Switzerland), who analyzed the fundamental processes of light harvesting and charge carrier generation and collection used by a molecular photovoltaic device applying concepts used by green plants and discussed the latest research advances in this field. E. W. Meijer (Eindhoven, The Netherlands) spoke about "The Non-covalent Synthesis of Function Supramolecular Systems," outlining intriguing prospects of molecular electronics, nanotechnology, biomaterials, and the aim to close the gap between synthetic and biological molecular systems. "Model Catalyst Design: A Perspective at the Atomic Level" was presented by H.J. Freund (Berlin, Germany), while L. Latos-Grazynski (Wroclaw, Poland) described "Aromaticity Switching in Porphyrinoids and Heteroporphyrinoids." Finally, Joshua Jortner (Tel Aviv, Israel) gave a lecture on "Chemical Dynamics at Extremes from Ultracold Systems to Table-top Nuclear Fusion." These topics exemplify the broad scope of the conference, which included roughly 1600 oral and poster presentations during the 5-day congress.

It has to be noted, however, that for those active in fostering metrology in chemistry or focusing on quality, transparency and reliability of measurement results, the offerings of the congress program were less fruitful; none of the various sessions or presentations was explicitly devoted to these subjects. The next EuCheMS Chemistry Congress will take place in Prague (Czech Republic) in August 2012 and may offer an opportunity to expand the scope of the congress in this direction. 